\title{
Validities of Fractional Order Derivatives in Literatures Such as Riemann-Liouville, Euler, Caputo and Grünwald- Letnikov
}

\author{
Ali KARCI
}

Software Engineering Department, İnönü University, Malatya, Turkey, (ali.karci@inonu.edu.tr)

\begin{abstract}
In this paper, it has been proven that it would be more accurate to accept Euler, RiemannLiouville, Caputo, and Grünwald-Letnikov methods as curve fitting or amplitude shifting methods without derivative definition. Since these derivative methods do not cause to shift extremum points of corresponding relations/functions to zero (the roots of relations/functions which are derived by taking fractional order derivative such as Euler, Riemann-Liouville, Caputo, and Grünwald-Letnikov methods).
\end{abstract}

Keywords - Fractional order derivatives, Grünwald-Letnikov derivative, Riemann-Liouville derivative, Caputo derivative

\section{Introduction}

The classical derivative was defined in time of Newton and Leipniz, and their derivative definitions and approaches were regarded as integer order derivative. There is non-integer order (fractional order) derivative definitions / approaches such as Euler, Riemann-Liouville, GrünwaldLetnikov and Caputo fractional order derivatives. Due to this case, this study focused on Euler, Riemann-Liouville, Grünwald-Letnikov and Caputo fractional order derivatives.

Isaac Newton defined the fundamentals of classical mechanics and these studies were collected in "Philosophiæ Naturalis Principia Mathematica". The main focus of this study is to define the rates of changes of functions (Newton, 1687). The first important and detailed work in differential calculus and differential geometry was done in (L'Hôpital, 1696; L'Hôpital, 1715).

The derivative of any function can be symbolized as follow like Leipniz, Lacroix, Newton

$$
\frac{d^{n}}{d x^{n}} f(x), n \in N, x \in R
$$

mhich means that it symbolizes $\mathrm{n}^{\text {th }}$ derivative of function $\mathrm{f}(\mathrm{x})$. Newtonian derivative can be regarded as integer order derivative (Fig.1), so, there should be non-integer order derivative.

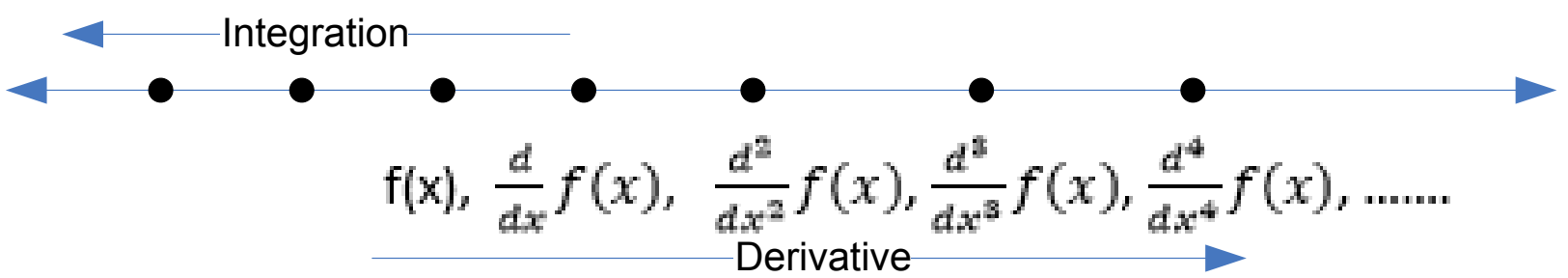

Figure 1. Integer order derivative. 
Some researchers considered the order of derivative as non-integer such as Euler, Riemann-Liouville, Grünwald-Letnikov and Caputo. The definitions of fractional order derivatives such as Euler, Riemann-Liouville, Grünwald-Letnikov and Caputo are given as follows:

Euler fractional order derivative (Das, 2011): $\frac{d^{\alpha}}{d x^{\alpha}} f(x)=\frac{\Gamma(\mathrm{n}+1)}{\Gamma(n+1-\alpha)} x^{n-\alpha}$ where $n \in \mathbb{N}, \alpha \in \mathbb{R}, \mathrm{f}(\mathrm{x})=\mathrm{x}^{\mathrm{n}}$.

Riemann-Liouville fractional order derivative (Das, 2011):

$$
{ }_{a} D_{x}^{\alpha} f(x)=\frac{1}{\Gamma(n-\alpha)}\left(\frac{d}{d x}\right)^{n} \int_{a}^{x} \frac{f(t)}{(x-t)^{\alpha-n+1}} d t, \quad(n-1) \leq \alpha<n
$$

Caputo fractional order derivative(Das, 2011):

$$
{ }_{a}^{C} D_{x}^{\alpha} f(x)=\frac{1}{\Gamma(n-\alpha)} \int_{a}^{x} \frac{f(t)}{(x-t)^{\alpha-n+1}} d t, \quad(n-1) \leq \alpha<n
$$

Grünwald-Letnikov fractional order derivative (Das, 2011):

$$
{ }_{a} D_{x}^{\alpha} f(x)=\lim _{h \rightarrow 0} \frac{1}{h^{\alpha}} \sum_{i=0}^{\left[\frac{t-a}{h}\right]}(-1)^{i}\left(\begin{array}{c}
\alpha \\
i
\end{array}\right) f(x-i h), \text { where }\left[\frac{t-a}{h}\right] \in \mathbb{Z}
$$

The aim of this study is to try to explain that most of the definitions of fractional derivatives given in the literature (except Karci's definition) are not valid based on the concept of derivative.

\section{Validities of Fractional Order Derivatives' Definitions}

There are some important axioms or postulates about integers or countable number of steps. The peano's axioms (postulates) are good examples for this concept (Hatcher, 1982). The Peano's axioms were written from (Hatcher, 1982), and Peano's axioms can be written in two different ways:

Peano's Axiom (Peano postulates): $\mathrm{S}$ is a set whose properties are:

a) $\mathrm{S}$ has a distinguished element which is called ' 1 '.

b) There exists a distinguished set map $\delta: S \rightarrow S$.

c) $\delta$ is one-to-one (injective).

d) There does not exist an element $\mathrm{s} \in \mathrm{S}$ such that $\delta(\mathrm{s})=1$.

e) $1 \in S$

f) Assume $R \subset S$ such that if $r \in R$, then $\delta(r) \in S$ ( $\delta(r)$ is successor of $r)$. Finally, $R=S$.

The Peano's axiom demonstrates that all step in this axiom should be an integer number, not a real number, successive events can be symbolized by using integer or natural numbers, not real numbers, i.e. the successive number of 0.1 is unknown. Peano's axioms (postulates) are very nice examples for this case; it is not a correct method in mathematics to accept integers order for derivative until the point where it works for you, and to accept order of derivative as real numbers after that point because it works for you. The same reasoning can be done for mathematical induction, since it requires steps. The mathematical induction is an applications of modus ponens successively many times. By using this idea, it can be verified that some fractional order derivative definitions have deficiencies (not sound and complete).

Euler Fractional Order Derivative: Assume that $\mathrm{f}(\mathrm{x})=\mathrm{x}^{\mathrm{n}}$, and its derivatives are

$$
\begin{gathered}
\frac{d}{d x} f(x)=n x^{n-1} \\
\frac{d^{2}}{d x^{2}} f(x)=n(n-1) x^{n-2}
\end{gathered}
$$




$$
\begin{gathered}
\frac{d^{3}}{d x^{3}} f(x)=n(n-1)(n-2) x^{n-3} \\
\frac{d^{4}}{d x^{4}} f(x)=n(n-1)(n-2)(n-3) x^{n-4} \\
\frac{d^{k}}{d x^{k}} f(x)=n(n-1)(n-2)(n-3) x^{n-k}
\end{gathered}
$$

$\mathrm{k}^{\text {th }}$ step of derivative is regarded as a real number and fractional order derivative is defined as follow

$$
\frac{d^{\alpha}}{d x^{\alpha}} f(x)=n(n-1)(n-2)(n-3) x^{n-\alpha}
$$

and coefficient of $x^{n-\alpha}$ is regarded as Gamma function such as $\Gamma(n+1)=n$ !, since the order of derivative is integer order up to $\mathrm{k}^{\text {th }}$ step, and $\mathrm{k}^{\text {th }}$ step order is regarded as real; this is not meaningful assumption in mathematics, since the concepts handled in mathematics should be based on concrete acceptances and evidences. At the first step, if the order of derivative is real and this case goes on until $\mathrm{k}^{\text {th }}$ step, the orders of derivative are $\alpha_{1}, \alpha_{2}, \alpha_{3}, \ldots, \alpha_{\mathrm{k}}$. The derivatives up to $\mathrm{k}^{\text {th }}$ step can be listed as follows

$$
\begin{aligned}
& \frac{d^{\alpha_{1}}}{d x^{\alpha_{1}}} f(x)=n x^{n-\alpha_{1}} \\
& \frac{d^{\alpha_{2}}}{d x^{\alpha_{2}}}\left(\frac{d^{\alpha_{1}}}{d x^{\alpha_{1}}} f(x)\right)=n\left(n-\alpha_{1}\right) x^{n-\left(\alpha_{1}+\alpha_{2}\right)} \\
& \frac{d^{\alpha_{3}}}{d x^{\alpha_{3}}}\left(\frac{d^{\alpha_{2}}}{d x^{\alpha_{2}}}\left(\frac{d^{\alpha_{1}}}{d x^{\alpha_{1}}} f(x)\right)\right)=n\left(n-\alpha_{1}\right)\left(n-\alpha_{2}\right) x^{n-\left(\alpha_{1}+\alpha_{2}+\alpha_{3}\right)} \\
& \frac{d^{\alpha_{4}}}{d x^{\alpha_{4}}}\left(\frac{d^{\alpha_{3}}}{d x^{\alpha_{3}}}\left(\frac{d^{\alpha_{2}}}{d x^{\alpha_{2}}}\left(\frac{d^{\alpha_{1}}}{d x^{\alpha_{1}}} f(x)\right)\right)\right)=n\left(n-\alpha_{1}\right)\left(n-\left(\alpha_{1}+\alpha_{2}\right)\right)\left(n-\left(\alpha_{1}+\alpha_{2}+\alpha_{3}\right)\right) x^{n-\sum_{i=1}^{4} \alpha_{i}} \\
& \frac{d^{\alpha_{k}}}{d x^{\alpha_{k}}}\left(\ldots \frac{d^{\alpha_{4}}}{d x^{\alpha_{4}}}\left(\frac{d^{\alpha_{3}}}{d x^{\alpha_{3}}}\left(\frac{d^{\alpha_{2}}}{d x^{\alpha_{2}}}\left(\frac{d^{\alpha_{1}}}{d x^{\alpha_{1}}} f(x)\right)\right)\right) \ldots\right)=n \prod_{i=1}^{k}\left(n-\sum_{j=1}^{i} \alpha_{j}\right) x^{n-\sum_{i=1}^{k} \alpha_{i}}
\end{aligned}
$$

At the $\mathrm{k}^{\text {th }}$ step, the term $x^{n-k}$ turns into $x^{n-\sum_{i=1}^{k} \alpha_{i}}$, and the coefficient of this term is not Gamma function; it is $n \prod_{i=1}^{k}\left(n-\sum_{j=1}^{i} \alpha_{j}\right)$. Both of these cases are the deficiencies of this method and they are the result of arbitrary assumption.

Riemann-Liouville Fractional Order Derivative: The Riemann-Liouville fractional order derivative is defined as follow

$$
{ }_{a} D_{x}^{\alpha} f(x)=\frac{1}{\Gamma(n-\alpha)}\left(\frac{d}{d x}\right)^{n} \int_{a}^{x} \frac{f(t)}{(x-t)^{\alpha-n+1}} d t, \quad(n-1) \leq \alpha<n
$$

The integration can be formulated as follow $(\alpha=1)$

$$
\begin{gathered}
{ }_{a} D_{x}^{-\alpha} f(x)=\lim _{n \rightarrow \infty}(h) \sum_{i=0}^{n} f(x-i h) \\
=\lim _{n \rightarrow \infty} \sum_{i=0}^{n} f(x-i h) \cdot h
\end{gathered}
$$




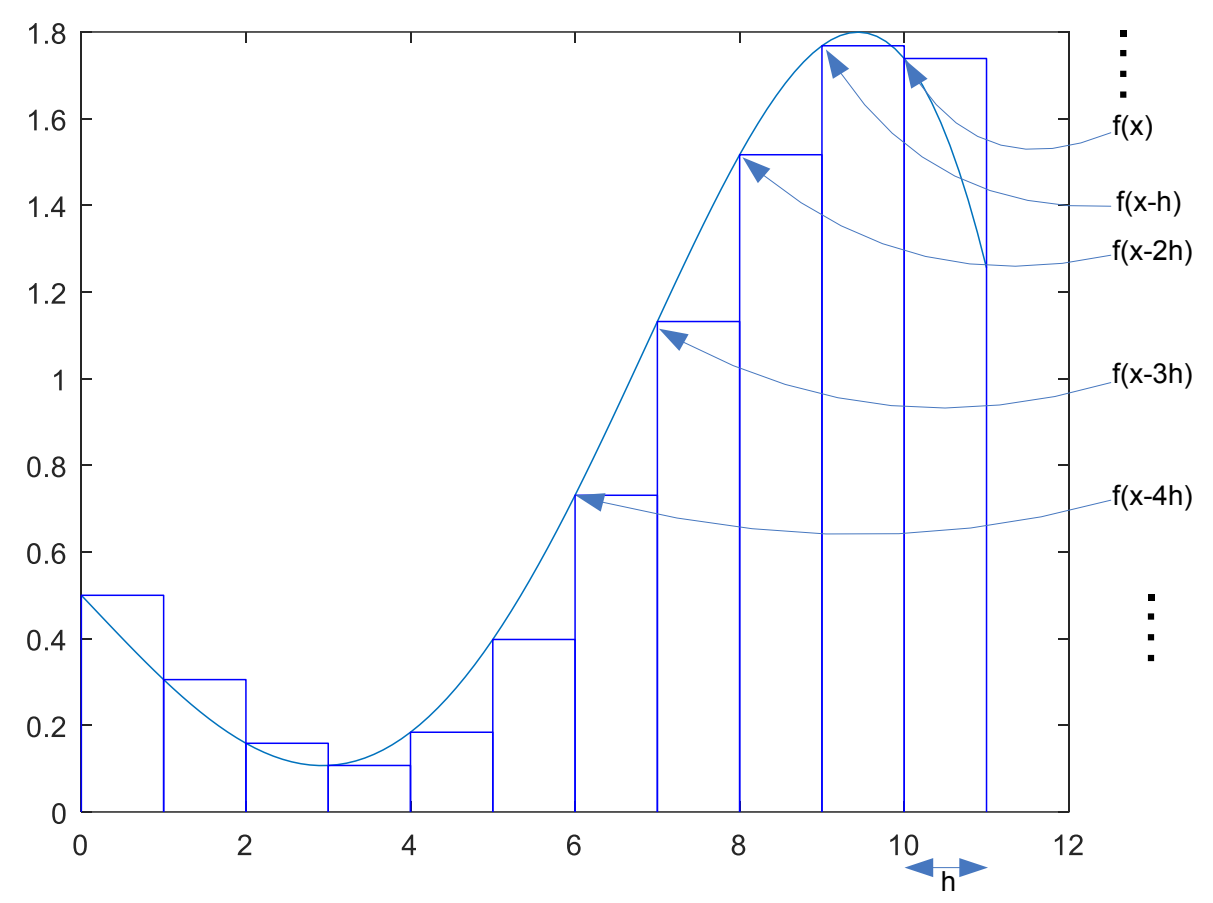

Figure 2. Riemann sum (Riemann integral when $\mathrm{h} \rightarrow 0$ )

Fig.2 illustrates this case (Riemann Integral), if $h=\Delta x=\frac{x-a}{n}$, then

If $u=x-t$ then $d x=-d u$

$$
{ }_{a} D_{x}^{-1} f(x)=\int_{0}^{x-a} f(x-t) d t
$$

When $\alpha=2$, then

$$
\begin{gathered}
{ }_{a} D_{x}^{-1} f(x)=\int_{x}^{a} f(u)(-d u) \\
{ }_{a} D_{x}{ }^{-1} f(x)=\int_{a}^{x} f(u) d u
\end{gathered}
$$

When $\alpha=3$, then

$$
{ }_{a} D_{x}{ }^{-2} f(x)=\int_{a}^{x}(x-u) f(u) d u
$$

When $\alpha=4$, then

$$
{ }_{a} D_{x}^{-3} f(x)=\frac{1}{2 !} \int_{a}^{x}(x-u)^{2} f(u) d u
$$

$$
\begin{gathered}
{ }_{a} D_{x}{ }^{-4} f(x)=\frac{1}{3 !} \int_{a}^{x}(x-u)^{3} f(u) d u \\
{ }_{a} D_{x}{ }^{-n} f(x)=\frac{1}{(n-1) !} \int_{a}^{x}(x-u)^{n-1} f(u) d u=\frac{1}{\Gamma(n)} \int_{a}^{x}(x-u)^{n-1} f(u) d u, \quad \text { when } \alpha=\mathrm{n} .
\end{gathered}
$$

When $\alpha \in \mathbb{R}$., this integration is called as Riemann-Liouville fractional integration (differintegration). All steps followed in above algebraic processes are integer, not real, the RiemannLiouville fractional integration was defined based in this algebraic processes. The Riemann-Liouville fractional order derivative is to take the derivative of this integration based on Newtonian derivative. That's why, all steps follow are integer, at any arbitrary step, this cannot be turned into real; this is the deficiencies of method and it needs proof based on real numbers, not integer and integer-based assumptions. The mathematical induction, modus ponens, Peano's axioms philosophy is also valid for the process of obtaining Riemann-Liouville fractional differintegration. 
Riemann-Liouville Fractional Order Derivative: The Riemann-Liouville fractional order derivative is

$$
{ }_{a}^{C} D_{x}^{\alpha} f(x)=\frac{1}{\Gamma(n-\alpha)} \int_{a}^{x} \frac{f(t)}{(x-t)^{\alpha-n+1}} d t, \quad(n-1) \leq \alpha<n
$$

The similar reasoning of Riemann-Liouville fractional order derivative can be done for Caputo fractional order derivative. The mathematical induction, modus ponens, Peano's axioms philosophy is also valid for the process of obtaining Riemann-Liouville fractional differintegration.

Grünwald-Letnikov fractional order derivative: The Grünwald-Letnikov fractional order derivative was obtained with similar manner like Riemann-Liouvile and Caputo fractional order derivatives.

The definition for derivative is

$$
{ }_{a} D_{x}^{\alpha} f(x)=\lim _{h \rightarrow 0} \frac{1}{h^{\alpha}} \sum_{i=0}^{\left[\frac{t-a}{h}\right]}(-1)^{i}\left(\begin{array}{c}
\alpha \\
i
\end{array}\right) f(x-i h), \text { where }\left[\frac{t-a}{h}\right] \in \mathbb{Z}
$$

$$
\begin{gathered}
\frac{d}{d x} f(x)=\lim _{h \rightarrow 0} \frac{f(x)-f(x-h)}{h} \\
\frac{d^{2}}{d x^{2}} f(x)=\lim _{h \rightarrow 0} \frac{f^{\prime(x)}-f^{\prime}(x-h)}{h}=\lim _{h \rightarrow 0} \frac{\frac{f(x)-f(x-h)}{h}-\frac{f(x-h)-f(x-2 h)}{h}}{h} \\
\frac{d^{2}}{d x^{2}} f(x)=\lim _{h \rightarrow 0} \frac{1}{h^{2}}(f(x)-2 f(x-h)+f(x-2 h)) \\
\frac{d^{3}}{d x^{3}} f(x)=\lim _{h \rightarrow 0} \frac{f^{\prime \prime}(x)-f^{\prime \prime}(x-h)}{h}=\lim _{h \rightarrow 0} \frac{\frac{f^{\prime}(x)-f^{\prime}(x-h)}{h}-\frac{f^{\prime}(x-h)-f^{\prime}(x-2 h)}{h}}{h} \\
\frac{d^{3}}{d x^{3}} f(x)=\lim _{h \rightarrow 0} \frac{1}{h^{3}}(f(x)-3 f(x-h)+3 f(x-2 h)+f(x-3 h)) \\
\frac{d^{n}}{d x^{n}} f(x)=\lim _{h \rightarrow 0}\left(\frac{1}{h}\right)^{n}\left(\sum_{i=0}^{n}(-1)^{i}\left(\begin{array}{l}
n \\
i
\end{array}\right) f(x-i h)\right)
\end{gathered}
$$

Grünwald-Letnikov fractional order derivative was defined by assumption of derivative order as real number. This is same as Euler, Riemann-Liouville and Caputo fractional derivatives. This is also some deficiencies of Grünwald-Letnikov fractional order derivative.

\section{Conclusions}

Peano's postulates are valid for natural numbers, mathematical induction is also valid for integer. Modus ponens is the origin of mathematical induction, so, the rules valid for Peano's postulates, mathematical induction, modus ponens do not necessarily apply to real numbers and do not necessarily valid for real numbers. The definition of fractional order derivative given in (Karci, 2013a; Karci, 2013b; Karci, 2015a; Karci, 2015b; Karc1 and Karc1, 2020) is valid, and it is not based on integer-based algebraic processes. The applications of this definition can be found in (Karc1, 2016; Karc1, 2017).

Euler, Riemann-Liouville, Caputo and Grünwald-Letnikov methods which are fractional order derivative definitions, are not actually derivative definitions, except that the step number is accepted as a real number in Newtonian derivative definition after some derivation steps. The handicaps of these methods are to accept derivation order as real number after some derivation steps and assume the coefficient as Gamma function. 


\section{References}

Newton, I. Philosophiæ Naturalis Principia Mathematica; Jussu Societatis Regiae ac Typis Joseph Streater. Prostat apud plures bibliopolas: London, UK, 1687.

L'Hôpital, G. Analyse des Infiniment Petits pour l'Intelligence des Lignes Courbes (Infinitesimal Calculus with Applications to Curved Lines); François Montalant: Paris, France, 1696.

L’Hôpital, G. Analyse des Infinement Petits; Relnk Books: Paris, France, 1715.

Das, S.,Functional fractional calculus, Springer, 2011.

Hatcher, W.S., The Logical Foundations of Mathematics, Pergamon Press, 1982.

Karc1, A.,'Kesirli Türev için Yapılan Tanımlamaların Eksiklikleri ve Yeni Yaklaşım”, TOK-2013 Turkish Automatic Control National Meeting and Exhibition, 2013a.

Karc1,A., "A New Approach for Fractional Order Derivative and Its Applications", Universal Journal of Engineering Sciences, Vol:1, pp: 110-117, 2013b.

Karc1, A., "Properties of Fractional Order Derivatives for Groups of Relations/Functions", Universal Journal of Engineering Sciences, vol:3, pp:39-45, 2015a.

Karc1,A., "The Properties of New Approach of Fractional Order Derivative", Journal of the Faculty of Engineering and Architecture of Gazi University, Vol.30, pp:487-501, 2015b.

Karc1, A.," Fractional order entropy New perspectives", Optik - International Journal for Light and Electron Optics, Vol:127, pp:9172-9177, 2016.

Karc1, A.," Malatya Functions: Symmetric Functions Obtained by Applying Fractional Order Derivative to Karc1 Entropy”, Anatolian Science Journal of Computer Sciences, Vol:2, pp:1-8, 2017.

Karc1, A.," Properties of Karc1's Fractional Order Derivative", Universal Journal of Engineering Science, Vol:7, pp:32-38, 2019.

Karc1, A., Karc1, Ş.," Discovering The Relationships between Fractional Order Derivatives and Complex Numbers", Anatolian Science - journal of Computer Science, Vol:5, pp:42-53, 2020. 